\title{
Long Term Exposure to Myrtucommulone-A Changes CD105 Expression and Differentiation Potential of Mesenchymal Stem Cells
}

\author{
Kenan Izgi ${ }^{2,3} \cdot$ Mehmet Fatih Sonmez $^{4} \cdot$ Halit Canatan ${ }^{1,3} \cdot$ Banu Iskender $^{1,3}$
}

Received: 19 February 2016/Revised: 22 March 2016/Accepted: 4 April 2016/Published online: 8 March 2017

(C) The Korean Tissue Engineering and Regenerative Medicine Society and Springer Science+Business Media Dordrecht 2017

\begin{abstract}
Mesenchymal stem cells (MSCs) represent a heterogeneous group of multipotent stem cells that could be found in various somatic tissues. MSCs are defined by molecular and functional features including spindle-shape morphology, adherence to plastic surfaces, expression of specific surface markers and differentiation potential to chondrocytes, adipocytes and osteocytes. The surface markers were proposed to affect the differentiation potential of MSCs by a limited number of studies. Endoglin (CD105) is defined to be a significant marker for osteogenic and chondrogenic differentiation ability of MSCs. Low CD105 expression is associated with increased osteogenic potential while high CD105 expression is correlated with strong chondrogenic potential. Myrtucommulone-A (MC-A) is an active compound with various biological effects on different cell types but its effect on MSC differentiation has not been described yet. In the present study we aimed at investigating the longterm effects of MC-A on hMSCs. MC-A-treatment reduced CD105 expression in distinct human mesenchymal stem cell (hMSC) lines and gave rise to $\mathrm{CD} 105^{\text {low }}$ population but did not change CD44, CD90 or CD73 expression. The decrease in CD105 expression reduced the chondrogenic potential of hMSCs subsequently while adipogenic or osteogenic differentiation was not affected dramatically. MC-A-treatment also suppressed the NF- $\mathrm{BB}$ p65 activation which might be responsible for the reduced chondrogenic potential. Our findings suggest that MC-A could be used to enrich CD $105^{\text {low }} \mathrm{hMSCs}$ without the need for cell sorting or changing culture conditions which could be utilised in targeted differentiation studies.
\end{abstract}

Keywords Human mesenchymal stem cells · CD105 - Myrtucommulone-A · Chondrogenic differentiation

Banu Iskender

banu.iskender@yahoo.com

Kenan Izgi

kenanizgi@erciyes.edu.tr

Mehmet Fatih Sonmez

mfsonmez@erciyes.edu.tr

Halit Canatan

halitcanatan@yahoo.com

1 Department of Medical Biology, Faculty of Medicine, Erciyes University, 38039 Melikgazi, Kayseri, Turkey

2 Department of Medical Biochemistry, Faculty of Medicine, Erciyes University, 38039 Melikgazi, Kayseri, Turkey

3 Betul-Ziya Eren Genome and Stem Cell Centre, Erciyes University, 38039 Melikgazi, Kayseri, Turkey

4 Department of Histology and Embryology, Faculty of Medicine, Erciyes University, 38039 Melikgazi, Kayseri, Turkey

\section{Introduction}

Mesenchymal stem cells (MSCs) represent the cell population bearing the multipotent ability in all somatic tissues. Their potential to differentiate into multiple lineages as well as their immunomodulatory properties render them significant tools for transplantation and cell-based therapies [1]. The minimum criteria to define human Mesenchymal Stem Cells (hMSCs) include adherence to plastic, expression of cell surface markers CD44, CD90, CD73, CD105 and lacking expression of CD14, CD34, CD45 and HLADR and the ability to differentiate into adipocytes, chondrocytes and osteocytes [2]. MSCs were first reported to be isolated from bone marrow but since then they have been isolated from various tissues, sharing the minimal criteria for hMSCs but also exhibiting distinct features [3, 4]. 
Endoglin (CD105) has been reported to be a significant marker for osteogenic and chondrogenic differentiation ability of MSCs [5, 6]. Low CD105 expression was found to be correlated with increased osteogenic differentiation $[6,7]$ whereas CD105 high fraction in MSCs were suggested to exhibit strong chondrogenic potential [8]. CD105 is a co-receptor for transforming growth factor $\beta 1$ and $\beta 3$ and its involvement in the regulation of osteogenic differentiation is not surprising as transforming growth factor beta (TGF $\beta$ ) signaling is known to suppress osteogenic differentiation and maturation [9]. Besides affecting multipotency of MSCs, endoglin expression has been reported to be variable depending on the culture conditions and culture time [10, 11].

Myrtucommulone-A (MC-A) is an active compound isolated from the leaves of myrtle [12]. Previously we have shown that MC-A-treatment decreased multipotency-associated marker expression in cancer cells [13] and shortterm MC-A-treatment reduced the cytokine expression in hMSCs [14]. In the present study we sought to determine the long-term effects of MC-A on the stemness of hMSCs and analysed the differentiation potential of hMSCs upon extended MC-A-treatment.

\section{Materials and methods}

\subsection{Cell culture}

Adipose-tissue derived hMSCs from 3 different sources were obtained from Erciyes University Betul-Ziya Eren Genome and Stem Cell Centre (Kayseri, Turkey). hMSCs were cultured in DMEM Low Glucose (Biological Industries) supplemented with 10\% FBS (Biochrom), 1\% penicilin-streptomycin and 1\% GlutaMax (Life Technologies) and incubated at $37{ }^{\circ} \mathrm{C}$ and $5 \% \mathrm{CO}_{2}$.

\subsection{Flow cytometry analysis}

hMSCs at passage 5 were treated with $5 \mu \mathrm{M}$ MC-A for 15 days. Flow cytometry analysis was performed on MC-A treated hMSCs and control cells for the specific cell surface antigens as described previously [13]. Briefly, cells were dissociated and resuspended in PBS+FBS at a concentration of $1 \times 10^{6}$ cells per condition. Antibody cocktails against CD73, CD90, CD44, CD105 and negative cocktails CD45, CD33, CD11b, CD19 and HLA-DR were added to samples and incubated for 30 minutes at dark at room temperature. The samples were washed and analyzed on BD FACS Canto A flow cytometer (BD Biosciences). FACS Diva v6.1.3 (BD Biosciences) software was used for data acquisition and analysis.

\subsection{Differentiation assays}

Adipogenic, chondrogenic and osteogenic differentiation protocols were conducted on control hMSCs and hMSCs pre-treated with MC-A for 10 days. Adipogenic, chondrogenic and osteogenic induction were performed according to manufacturer's instructions and as described earlier [13]. During the differentiation protocols low-dose $(1 \mu \mathrm{M}) \mathrm{MC}$-A-treatment was sustained at every medium change. Samples from day 7, day 14 and day 21 were collected for the analysis of adipogenic, chondrogenic and osteogenic markers.

\subsection{Immunocytochemistry}

After the differentiation protocols cells were evaluated for the expression of adipogenic, chondrogenic and osteogenic markers. The cells were fixed with $4 \%$ (w/v) paraformaldehyde, blocked with PBS containing 5\% BSA and $\% \quad 0.3$ triton-x-100 and stained. Primary antibodies against FAB4 and osteocalcin were used at $1 \mu \mathrm{g} / \mathrm{ml}$ concentration (all from R\&D Systems). Alexa Fluor-488 conjugated anti Ig (Invitrogen) was used as a secondary antibody. Chondrogenic pellets were sectioned and stained according to the protocol described by Solchaga et al. [15].

\subsection{Quantitative real-time PCR}

To validate the chondrogenic differentiation potential of hMSCs the mRNA levels of chondrogenic genes were analysed using quantitative real-time PCR as described previously [13]. The qPCR primers used for detecting gene expression were as follows: Collagen II (GenBank XM_011537935.1) F: GGCAATAGCAGGTTCACGTACA R: CGATAACAGTC TTGCCCCACTT; Collagen X (GenBank NM_000493.3) F: CAAGGCACCATCTCCAGGAA R: AAAGGGTATTTG TGGCAGCATATT; SOX5 (GenBank XM_011520843.1) F: ATCCCAACTACCATGGCAGCT R: TGCAGTTGGAGTG GGCCTA; Aggrecan (GenBank XM_011521314.1) F: TC GAGGACAGCGAGGCC R: TCGAGGGTGTAGCGTGTA GAGA; SOX9 (GenBank NM_000346.3) F: GACTTCCGC GACGTGGAC R: GTTGGGCGGCAGGTACTG; GAPDH (GenBank NM_001289746.1) F: ATGGGGAAGGTGAAGG TCG R: TAAAAGCAGCCCTGGTGACC.

\subsection{Western blotting}

Cell lysates were collected from three individual hMSC lines before treatment and after treatment with $5 \mu \mathrm{M} \mathrm{MC}$ A for 5 days. $10 \mu \mathrm{g}$ protein from each sample was loaded on Bis-Tris gradient gels (Invitrogen), seperated and transferred electrophoretically on to nitrocellulose membranes (Bio-Rad). Membranes were blocked and incubated 
with primary antibodies against CD105 (endoglin) (Cell Signaling), CD44 (R\&D Systems), phospho-NF-кB p65

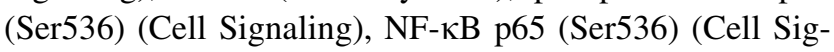
naling), Smad2 (Cell Signaling). GAPDH was used as a loading control. A horseradish peroxidase-conjugated IgG was used to detect signal which then was visualised by enhanced chemiluminescence.

\subsection{Statistical analysis}

The unpaired Student's t-test was used for analysis. Data in bar graphs indicate the mean $\pm \mathrm{SD}$, and statistical significance is expressed as follows: $* p<0.001$; ** $p<0.05$.

\section{Results}

\subsection{MC-A reduces CD105 expression in hMSCs}

To check whether MC-A-treatment has an effect on hMSC proliferation two individual hMSC lines were treated with increasing doses of MC-A $(1,2.5,5$ and $10 \mu \mathrm{M})$ and proliferation assay after $24 \mathrm{~h}$ and $48 \mathrm{~h}$ was performed. Except the highest dose $(10 \mu \mathrm{M})$, other doses did not significantly inhibit hMSC proliferation (Fig. 1A). The highest dose was chosen which did not affect hMSC proliferation $(5 \mu \mathrm{M})$ for further analysis. hMSCs were treated with $5 \mu \mathrm{M}$ MC-A for 15 days and analysed by flow cytometry. The expression level of CD105 was reduced by $70.7 \%$ in MC-A treated hMSCs in comparison to the parental hMSCs. Other cell surface markers, CD90, CD44 and CD73 was not dramatically affected from MC-A-treatment (Fig. 1B). Immunocytochemistry also confirmed the decreased expression of CD105 in MC-A treated hMSCs while CD44 expression was not affected (Fig. 1C).

\subsection{MC-A-treatment in hMSCs decreased the chondrogenic differentiation ability but did not change adipogenic or osteogenic differentiation}

Since a dramatic decrease was detected in one of the stemness markers (CD105), the differentiation ability of hMSC was assessed upon MC-A conditioning. First the hMSCs were exposed to MC-A for 10 days then adipogenic, osteogenic and chondrogenic differentiation was induced. MC-A-treatment was sustained during the differentiation protocols in comparison to the untreated control hMSCs. The decrease in CD105 expression induced by MC-A-treatment did not dramatically affect the adipogenic differentiation of hMSCS (Fig. 2A). MC-A-treatment changed the morphology of osteo-differentiated cells compared to the controls but the efficiency of osteogenic differentiation was not measured although MC-A treated hMSCs seemed to deposit osteocalcin earlier than the control group (Fig. 2B). After 2 weeks of chondrogenic differentiation, control and $\mathrm{MC}-\mathrm{A}$ treated condrogenic micromasses were sectioned and analysed. MC-A treated pellets exhibited smaller moprhology as well as weaker safranin $\mathrm{O}$ staining for aggrecans compared to the control group. Toluidine blue staining revealed metachromasia due to the proteoglycan content in the control group while MCA treated micromasses failed to exhibit. Similarly, Masson's Trichrome staining indicated the presence of collagen (blue) in control group which demonstrated weaker staining in MC-A treated micromasses (Fig. 3A). The expression levels of genes associated with chondrogenic differentiation were further evaluated in the differentiated hMSCs. The expression levels of SOX9, SOX5, aggrecan and collagen II under control differentiation conditions for 7 days were significantly higher than the MC-A treated micromasses. Collagen $\mathrm{X}$ which is known to be a marker of chondrocyte hypertrophy and replacement by bone during chondrogenesis [16] was determined to be significantly upregulated in MC-A treated samples (Fig. 3B).

\subsection{Down-regulation of NF-кB activity upon MC-A- treatment might account for the reduced chondrogenic differentiation ability}

Protein levels of undifferentiated marker expression CD44 and CD105 were evaluated for three independent hMSC lines, treated or not treated with MC-A. MC-A-treatment in 3 hMSC lines reduced CD105 expression but did not significantly alter CD44 expression consistent with the flow cytometry and immunofluorescence data (Fig. 4A). MC-Atreatment was also shown to reduce total protein level of SMAD2 which is a well-known signalling intermediator of TGF $\beta$. Moreover, phosphorylation state of NF- $\kappa \mathrm{B}$ p65 was dramatically affected from MC-A-treatment in all hMSC lines which might suggest involvement of $\mathrm{NF}-\kappa \mathrm{B}$ pathway as well as TGF $\beta$ signaling in reduced chondrogenic differentiation ability of hMSCs (Fig. 4A, B).

\section{Discussion}

The aim of the present study was to characterise the longterm effects of MC-A on the cell surface marker expression of hMSCs and in vitro differentiation potential. Long-term treatment with MC-A specifically and significantly reduced the cell surface marker CD105 expression. CD105 ${ }^{\text {low }}$ hMSCs induced by MC-A-treatment exhibited limited chondrogenic differentiation potential compared to the control hMSCs but adipogenic and osteogenic differentiation potential was not impaired. 
A
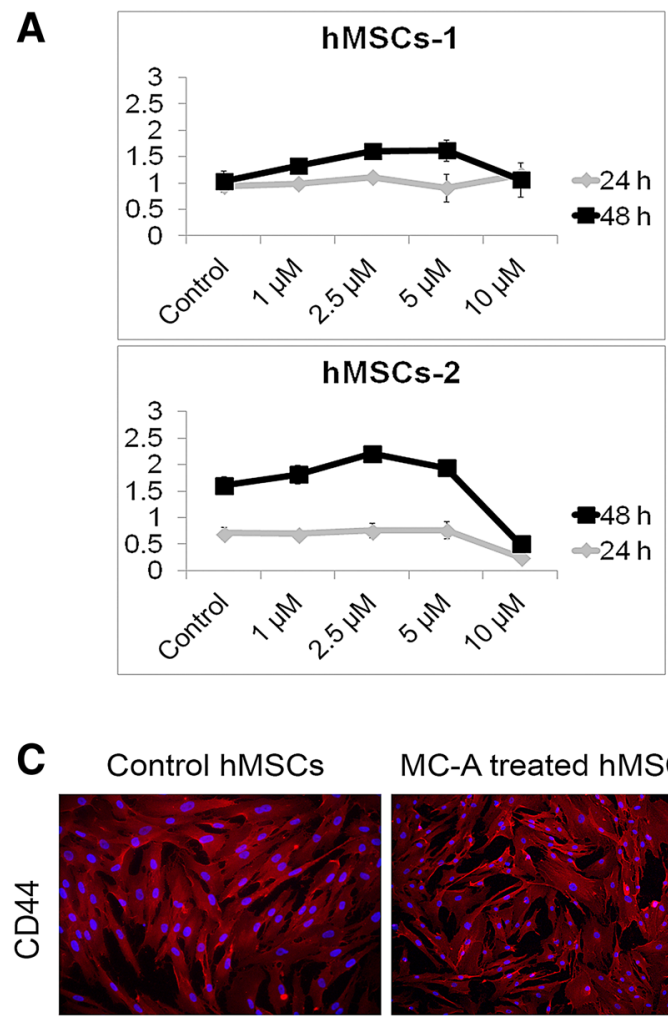

MC-A treated hMSCs
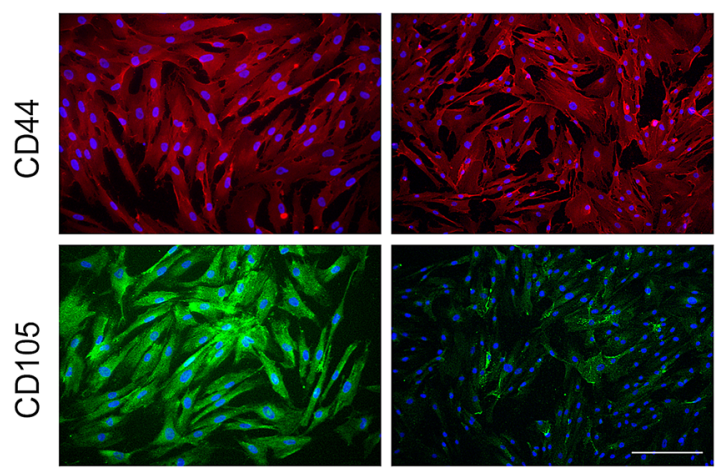

Fig. 1 MC-A reduced CD105 expression in hMSCs without affecting cell proliferation. A Two different hMSC lines incubated with various concentrations of MC-A $(1,2.5,5,10 \mu \mathrm{M})$ for 24 and $48 \mathrm{~h}$ and cell proliferation was measured by BrdU assay. MC-A did not significantly reduce or inhibit hMSC proliferation. B Flow cytometry analysis revealed that MC-A-treatment resulted in a decrease in

It is well-established that the expression of stemness markers varies depending on the MSC source (bone marrow, adipose, umblical cord, etc.), culture conditions (serum depletion) or culture time $[2,7,10,17]$. The variability does not only occur in additional stemness marker expression but also in differentiation potential of MSCs derived from distinct sources which might suggest the involvement of stemness markers in the regulation of differentiation potential [18-20]. For instance, inhibition of CD73 expression was found to be related with the increase in chondrogenic matrix deposition [21] and CD29+/ CD90+ sub-population of bone marrow stem cells exhibited reduced adipogenic and osteogenic differentiation [22].

The studies on the roles of stemness-related markers in differentiation are currently very limited but the role of CD105 on MSC differentiation is relatively well-studied.

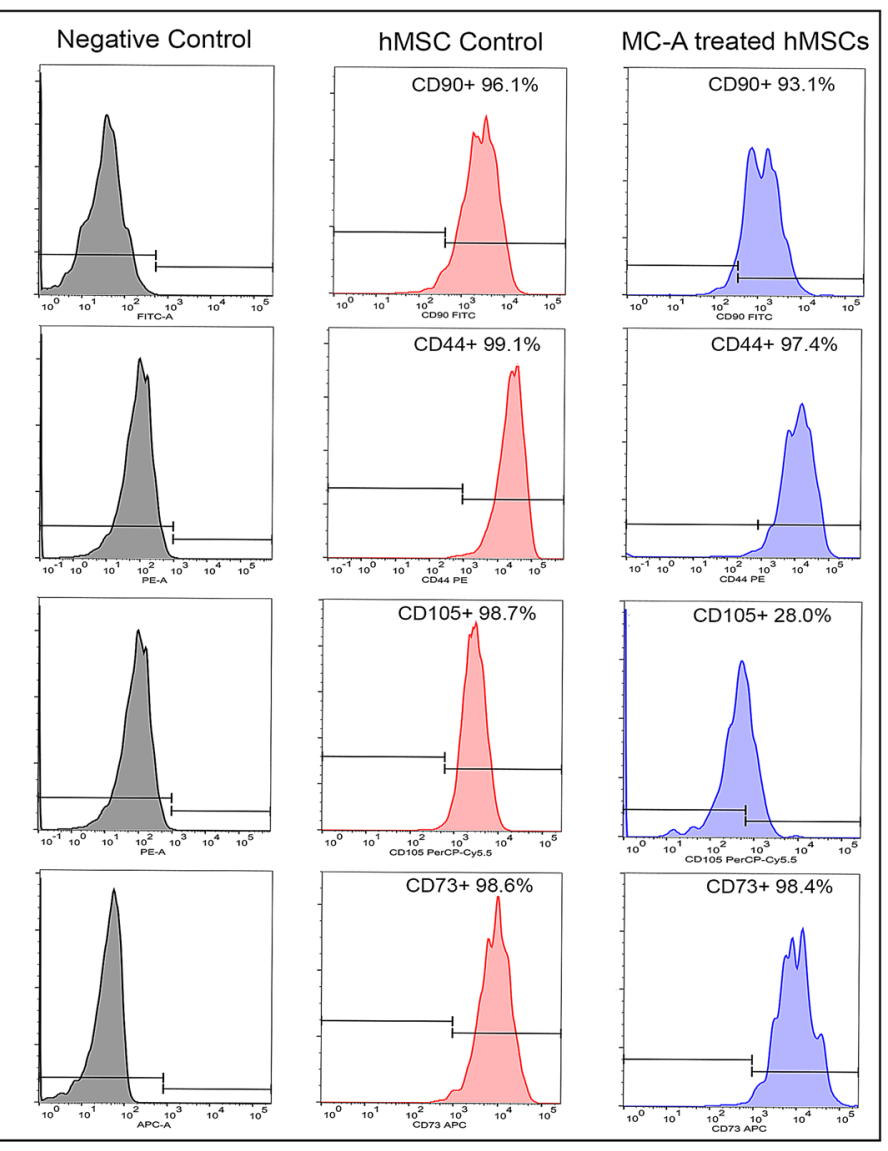

CD105 expression while other positive markers CD90, CD44, CD73 levels were not dramatically affected. C Immunofluorescence analysis confirmed reduced expression of CD105 in MC-A treated hMSCs. MC-A: myrtucommulone-A, BrdU: bromodeoxyuridine, CD105: endoglin, hMSCs: human mesenchymal stem cells

CD105 is known to possess biological functions in angiogenesis, survival of endothelial cells and development of cardiovascular system [23]. Positive expression of CD105 is one of the selection criteria for MSCs and the decrease in CD105 expression is associated with MSC differentiation [24]. Endoglin (CD105) is a co-receptor for TGF $\beta$ and is known to be expressed in human chondrocytes $[25,26]$. CD105 binds to TGF $\beta$ type II receptors thereby acts as a key factor in the regulation of MSC response to TGF $\beta 1$ and TGF $\beta 3$ [26]. Since TGF $\beta$ signaling is fundamental in chondrogenic differentiation of MSCs, lack of CD105 expression could be linked to the decreased chondrogenic differentiation ability of MSCs [27]. Indeed, CD105 ${ }^{\text {high }}$ MSCs were shown to differentiate more effectively into chondrocytes compared to CD105 ${ }^{\text {low }}$ MSCs [28]. Moreover, hMSCs expressing low CD105 exhibited increased osteogenic gene expression and were more prone to 
A
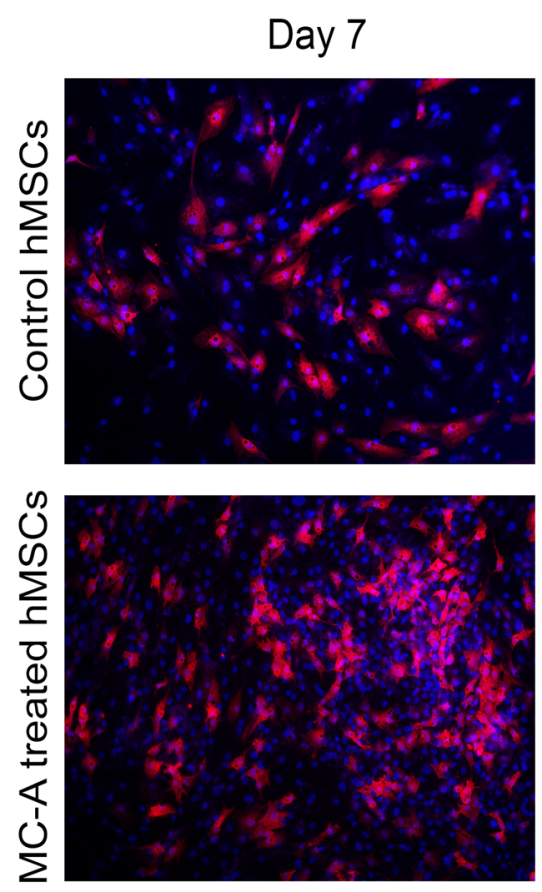

B
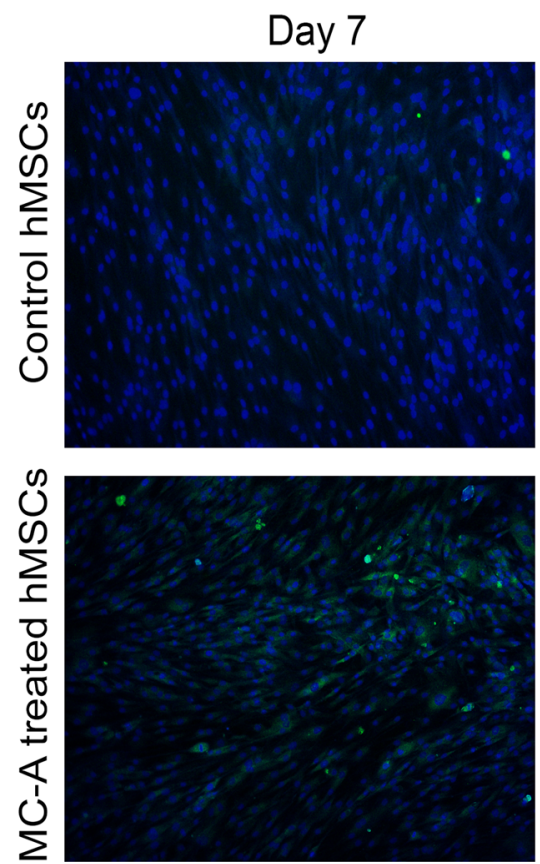

Fig. 2 MC-A-treatment did not change adipogenic or osteogenic differentiation potential of hMSCs. A hMSCs were pre-treated with MC-A or control MSCs were cultured in adipogenic induction medium for 21 days and analysed for the expression of adipogenic marker FABP4 by immunofluorescence at day 7, 14 and 21 of differentiation. B hMSCs were pre-treated with MC-A or control MSCs were cultured in osteogenic induction medium for 21 days and

\section{Fatty Acid-Binding Protein 4}
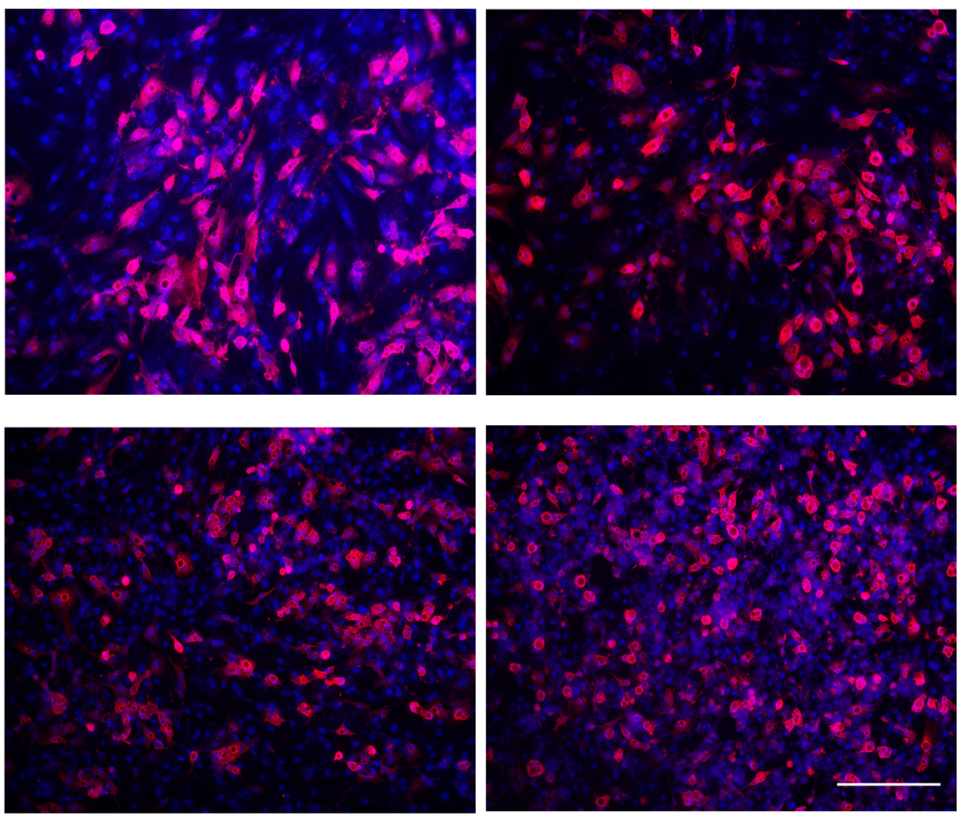

\section{Osteocalcin}

Day 14
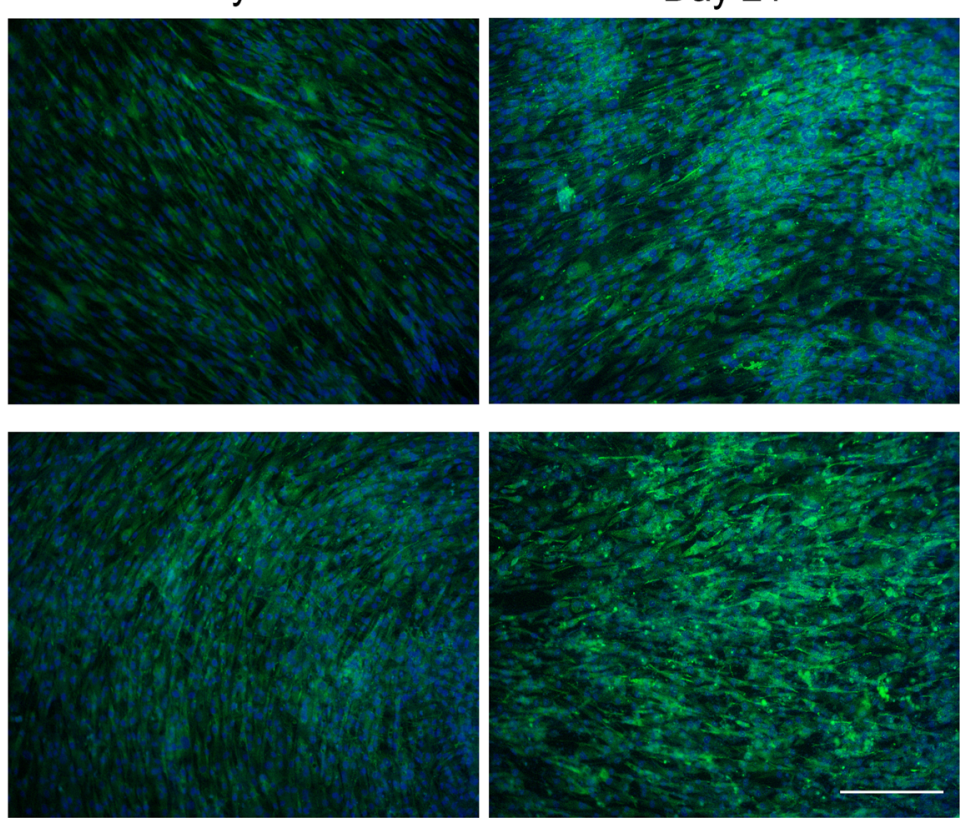

analysed for the expression of Osteocalcin by immunofluorescence at day 7,14 and 21 of differentiation. Cell nuclei were stained with DAPI. Scale bar represent $100 \mu \mathrm{m}$. MC-A: myrtucommulone-A, hMSCs: human mesenchymal stem cells, MSCs: mesenchymal stem cells, FABP4: fatty acid-binding protein 4 , DAPI: 4',6-diamidino-2phenylindole 

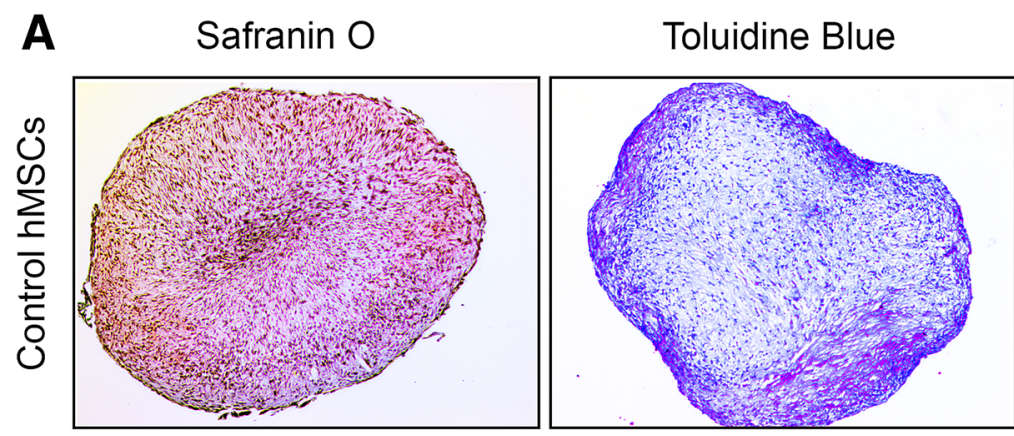

\section{Masson's Trichrome}
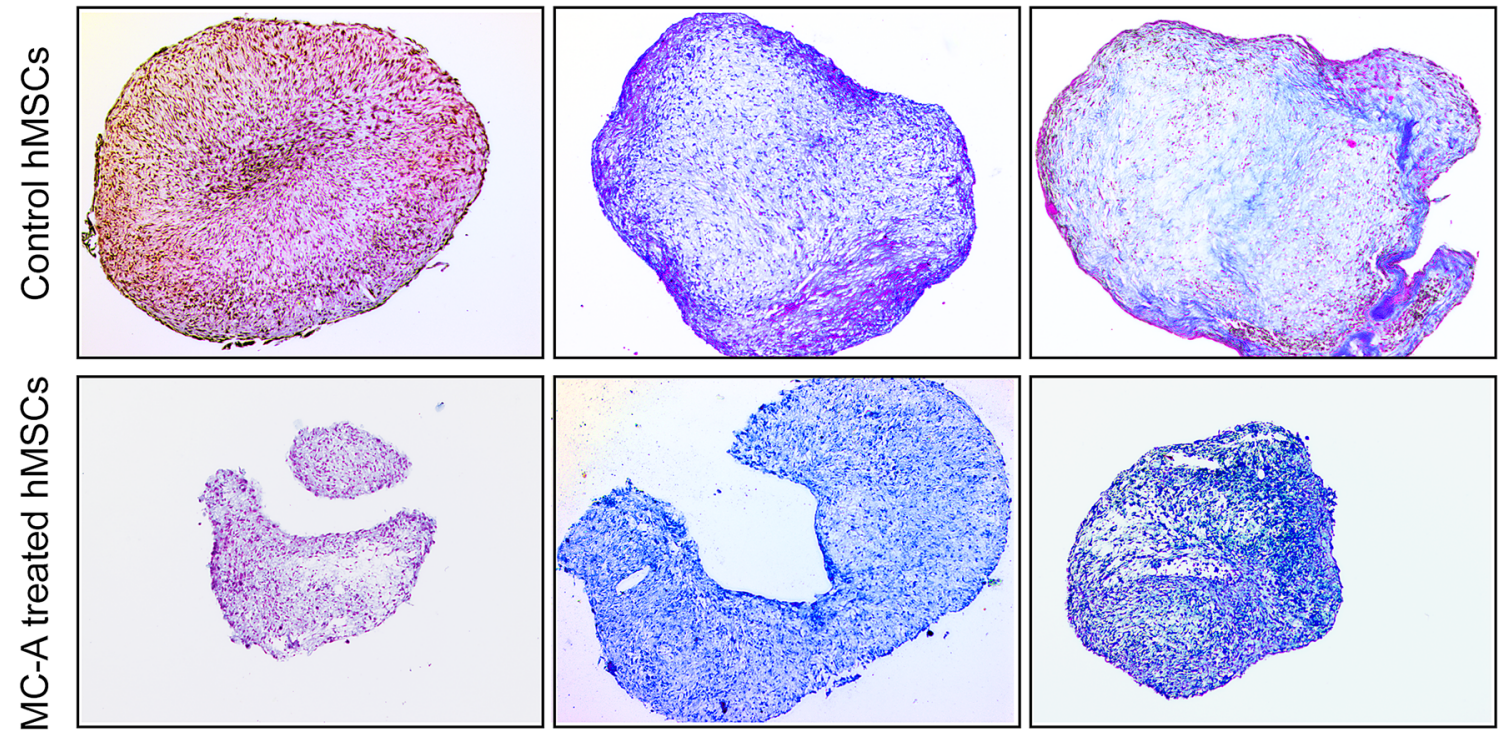

B

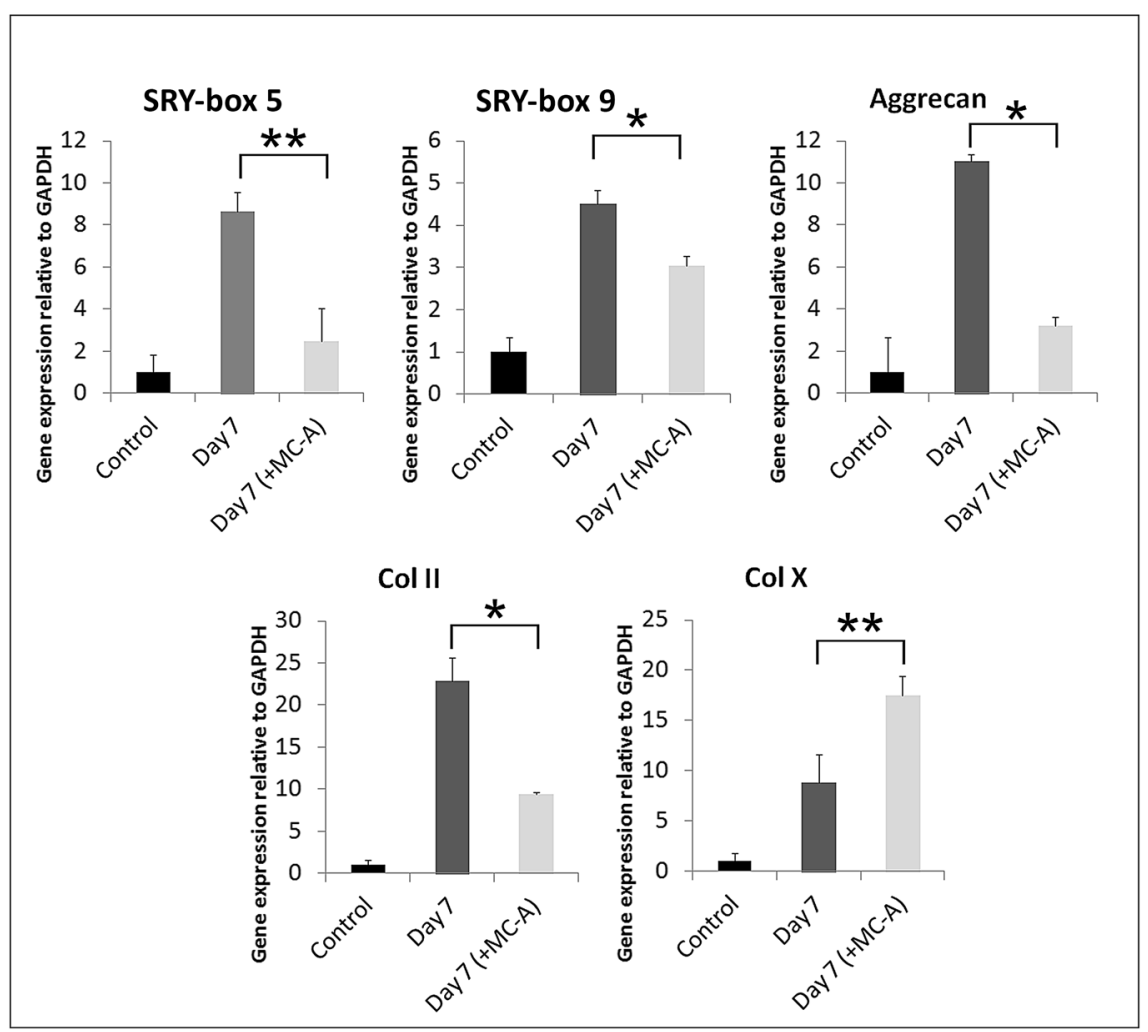

Fig. 3 MC-A-treatment reduced the chondrogenic differentiation potential of hMSCs. A hMSCs were pre-treated with MC-A or control MSCs were cultured in chondrogenic induction medium for 21 days and analysed for proteoglycan and collagen content by Safranin O, Toluidine Blue and Masson's Trichrome staining. MC-A treated chondrogenic micromasses exhibited reduced pellet size poor expression of proteoglycan/collagen. B Relative mRNA expressions of key factors involved in chondrogenic differentiation in MC-A treated hMSCs or untreated controls after 7 days of differentiation. Untreated hMSC controls exhibited significant upregulation of chondrogenic markers SOX5, SOX9, Aggrecan, Collagen II while MC-A treated samples expressed high levels of Collagen X. Data are expressed as the mean $\pm \mathrm{SD} ; * p<0.001$, ** $p<0.05$. MC-A: myrtucommulone-A, hMSCs: human mesenchymal stem cells, MSCs: mesenchymal stem cells, mRNA: messenger ribonucleic acid, SOX: SRY-box 
A

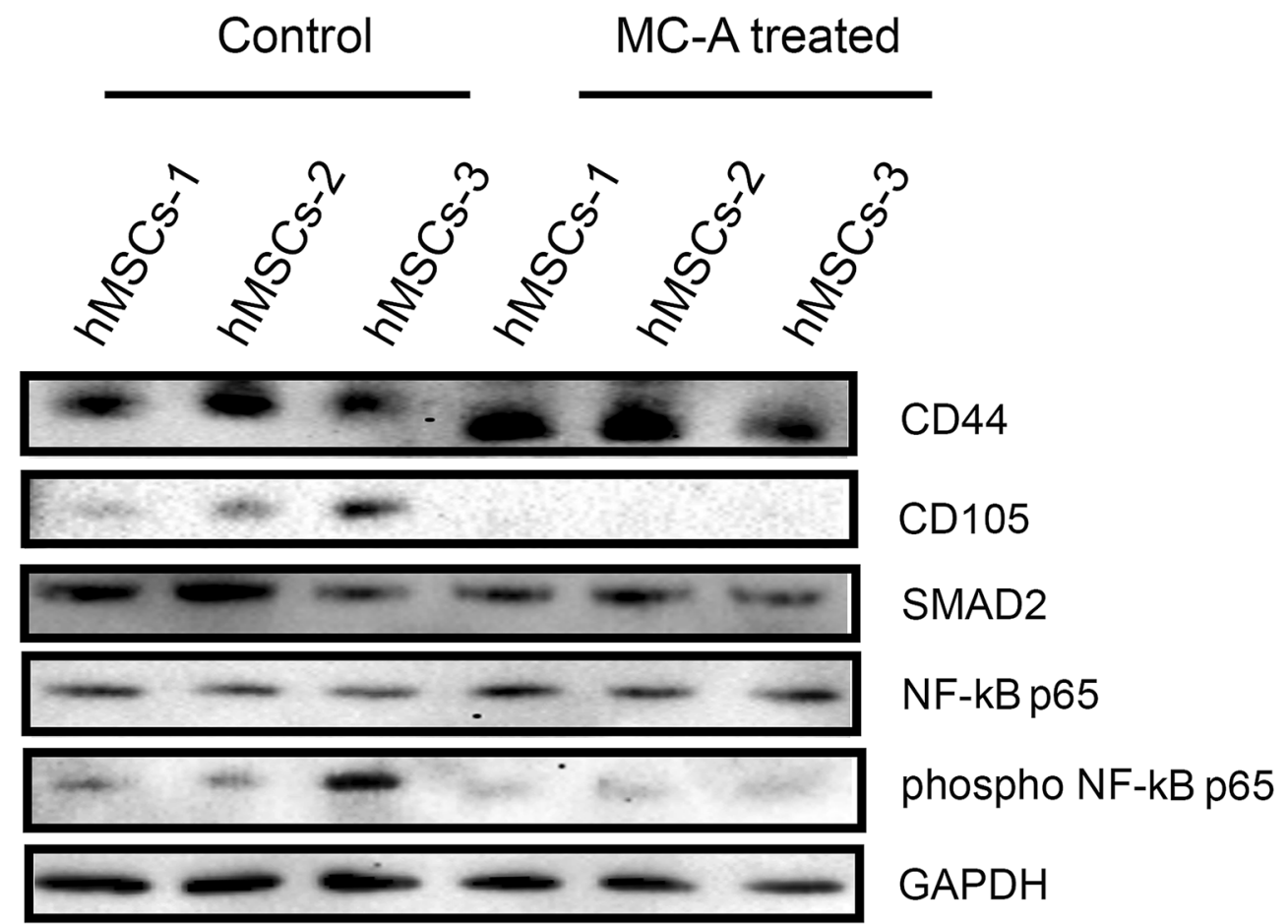

B
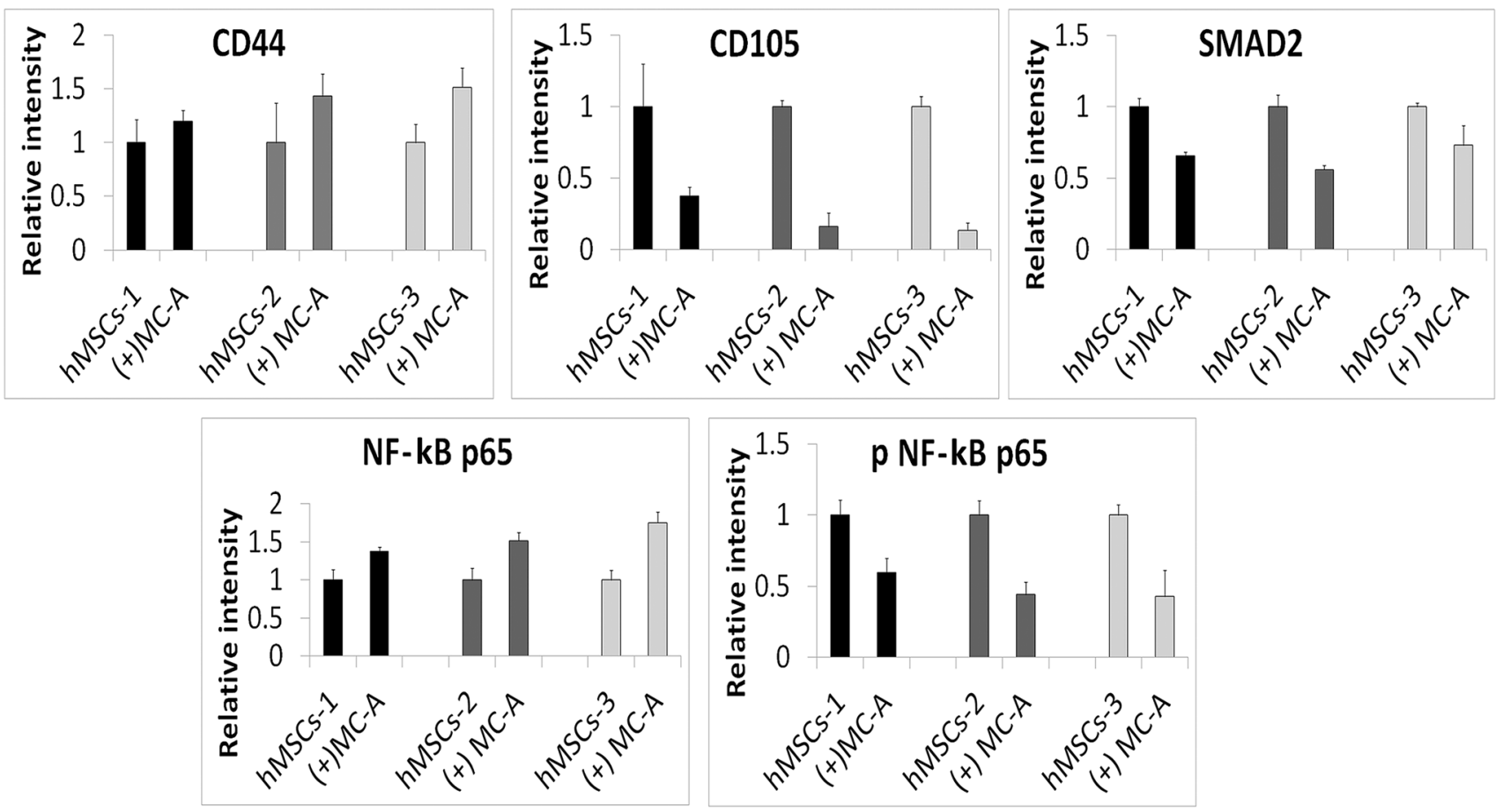

Fig. 4 MC-A induced changes in protein expression in three different hMSC lines. A hMSCs treated with MC-A demonstrated stable expression of CD44 while CD105 expression was inhibited in all cell lines consistent with flow cytometry analysis. Total protein level of SMAD2 was decreased in MC-A treated hMSCs. MC-A-treatment was also shown to suppress NF-kB p65 activation which is known to involve in chondorgenic differentiation. B The relative intensity of protein levels are expressed as bar graphs. GAPDH is used as a loading control and molecular weight is indicated in $\mathrm{kDa}$. MC-A: myrtucommulone-A, hMSCs: human mesenchymal stem cells, SMAD2: mothers against decapentaplegic homolog 2, GAPDH: glyceraldehyde 3-phosphate dehydrogenase 
osteogenic differentiation [6, 29]. In agreement with these studies, the present study revealed that $\mathrm{CD} 105^{\text {low }}$ population of hMSCs induced by long-term MC-A-treatment exhibited reduced chondrogenic differentiation potential. MC-A-treatment did not interfere with adipogenic or osteogenic differentiation potential of hMSCs but since $\mathrm{CD} 105^{\text {low }}$ population have been shown to enhance osteogenic differentiation, MC-A's effect on osteogenic potential could be further studied.

By suppressing TGF $\beta$ co-receptor CD105, MC-A-treatment decreased the total protein level of SMAD2 in three different hMSC lines. Interestingly, MC-A-treatment also suppressed NF-kB p65 phosphorylation. Previous studies suggested contradicting results regarding involvement of pathway in chondrogenic differentiation of hMSCs. Sirtuin-1 was reported to enhance chondrogenic differentiation of MSCs through inhibition of NF-KB pathway and activation of SOX9 [30]. Similarly, curcumin mediated inhibition of NF- $\mathrm{KB}$ facilitated the chondrogenic differentiation potential of hMSCs [31]. In contrast, early chondrogenic differentiation of MSCs was shown to be enhanced by transient activation of NF- $\mathrm{KB}$ p65 mediated signaling which in turn increased the chondrogenic marker expression [32]. Supporting the latter, the findings of the present study also demonstrated that chondrogenic differentiation potential of hMSCs was reduced upon MC-A-treatment which might be due to the supression of NF-kB activation. Further confirmation is necessary in order to elucidate the role of NF$\kappa \mathrm{B}$ in chondrogenic differentiation of hMSCs.

Taken together, we here proposed that MC-A-treatment could enrich CD $105^{\text {low }}$ hMSC population without the need of cell sorting or change in culture conditions like serum deprivation or growth factor stimulation. The results of this study clearly shows that MC-A could attenuate chondrogenic differentiation while leading the osteogenic or adipogenic differentiation of hMSCs. The enrichment method for $\mathrm{CD} 105^{\text {low }} \mathrm{hMSC}$ could be utilised in targeted differentiation of hMSC for cell-based regeneration studies.

Acknowledgements This study was supported by the grant from the The Scientific and Technological Research Council of Turkey (No. 115S042).

\section{Compliance with ethical standards}

Conflict of interest The authors declare no conflicts of interest.

Ethical statement There were no animal experiments carried out for this article.

\section{References}

1. Spencer ND, Gimble JM, Lopez MJ. Mesenchymal stromal cells: past, present, and future. Vet Surg. 2011;40:129-39.

2. Dominici M, Le Blanc K, Mueller I, Slaper-Cortenbach I, Marini F, Krause D, et al. Minimal criteria for defining multipotent mesenchymal stromal cells. The International Society for Cellular Therapy position statement. Cytotherapy. 2006;8:315-7.

3. Pittenger MF, Mackay AM, Beck SC, Jaiswal RK, Douglas R, Mosca JD, et al. Multilineage potential of adult human mesenchymal stem cells. Science. 1999;284:143-7.

4. Ullah I, Subbarao RB, Rho GJ. Human mesenchymal stem cells-current trends and future prospective. Biosci Rep. 2015;35:e00191.

5. Beyth S, Borovsky Z, Mevorach D, Liebergall M, Gazit Z, Aslan $\mathrm{H}$, et al. Human mesenchymal stem cells alter antigen-presenting cell maturation and induce T-cell unresponsiveness. Blood. 2005;105:2214-9.

6. Levi B, Wan DC, Glotzbach JP, Hyun J, Januszyk M, Montoro D, et al. CD105 protein depletion enhances human adipose-derived stromal cell osteogenesis through reduction of transforming growth factor beta1 (TGF-beta1) signaling. J Biol Chem. 2011;286:39497-509.

7. Anderson $\mathrm{P}$, Carrillo-Galvez AB, Garcia-Perez A, Cobo M, Martin F. CD105 (endoglin)-negative murine mesenchymal stromal cells define a new multipotent subpopulation with distinct differentiation and immunomodulatory capacities. PLoS ONE. 2013;8:e76979.

8. Qi J, Chen A, You H, Li K, Zhang D, Guo F. Proliferation and chondrogenic differentiation of CD105-positive enriched rat synovium-derived mesenchymal stem cells in three-dimensional porous scaffolds. Biomed Mater. 2011;6:015006.

9. Maeda S, Hayashi M, Komiya S, Imamura T, Miyazono K. Endogenous TGF-beta signaling suppresses maturation of osteoblastic mesenchymal cells. EMBO J. 2004;23:552-63.

10. Mark P, Kleinsorge M, Gaebel R, Lux CA, Toelk A, Pittermann $\mathrm{E}$, et al. Human mesenchymal stem cells display reduced expression of CD105 after culture in serum-free medium. Stem Cells Int. 2013;2013:698076.

11. Jiang T, Liu W, Lv X, Sun H, Zhang L, Liu Y, et al. Potent in vitro chondrogenesis of CD105 enriched human adiposederived stem cells. Biomaterials. 2010;31:3564-71.

12. Muller H, Paul M, Hartmann D, Huch V, Blaesius D, Koeberle A, et al. Total synthesis of myrtucommulone A. Angew Chem Int Ed Engl. 2010;49:2045-9.

13. Iskender B, Izgi K, Karaca H, Canatan H. Myrtucommulone-A treatment decreases pluripotency- and multipotency-associated marker expression in bladder cancer cell line HTB-9. J Nat Med. 2015;69:543-54.

14. Iskender B, Izgi K, Sakalar C, Canatan H (2015) Priming hMSCs with a putative anti-cancer compound, myrtucommulone-a: a way to harness hMSC cytokine expression via modulating PI3K/Akt pathway? Tumour Biol. 2016;37:1967-81.

15. Solchaga LA, Penick KJ, Welter JF. Chondrogenic differentiation of bone marrow-derived mesenchymal stem cells: tips and tricks. Methods Mol Biol. 2011;698:253-78.

16. Shen $G$. The role of type $X$ collagen in facilitating and regulating endochondral ossification of articular cartilage. Orthod Craniofac Res. 2005;8:11-7.

17. Wang D, Chen R, Zhong X, Fan Y, Lai W, Sun X. Levels of CD105 cells increase and cell proliferation decreases during S-phase arrest of amniotic fluid cells in long-term culture. Exp Ther Med. 2014;8:1604-10.

18. Shetty P, Cooper K, Viswanathan C. Comparison of proliferative and multilineage differentiation potentials of cord matrix, cord blood, and bone marrow mesenchymal stem cells. Asian J Transfus Sci. 2010;4:14-24.

19. Torensma R, Prins HJ, Schrama E, Verwiel ET, Martens AC, Roelofs H, et al. The impact of cell source, culture methodology, culture location, and individual donors on gene expression profiles of bone marrow-derived and adipose-derived stromal cells. Stem Cells Dev. 2013;22:1086-96. 
20. Zhu X, Shi W, Tai W, Liu F. The comparition of biological characteristics and multilineage differentiation of bone marrow and adipose derived Mesenchymal stem cells. Cell Tissue Res. 2012;350:277-87.

21. Ode A, Schoon J, Kurtz A, Gaetjen M, Ode JE, Geissler S, et al. CD73/5'-ecto-nucleotidase acts as a regulatory factor in osteo-/chondrogenic differentiation of mechanically stimulated mesenchymal stromal cells. Eur Cell Mater. 2013;25:37-47.

22. Davies OG, Cooper PR, Shelton RM, Smith AJ, Scheven BA. Isolation of adipose and bone marrow mesenchymal stem cells using CD29 and CD90 modifies their capacity for osteogenic and adipogenic differentiation. J Tissue Eng. 2015;6:20417314 15592356.

23. Nassiri F, Cusimano MD, Scheithauer BW, Rotondo F, Fazio A, Yousef GM, et al. Endoglin (CD105): a review of its role in angiogenesis and tumor diagnosis, progression and therapy. Anticancer Res. 2011;31:2283-90.

24. Jin HJ, Park SK, Oh W, Yang YS, Kim SW, Choi SJ. Downregulation of CD105 is associated with multi-lineage differentiation in human umbilical cord blood-derived mesenchymal stem cells. Biochem Biophys Res Commun. 2009;381:676-81.

25. Parker WL, Goldring MB, Philip A. Endoglin is expressed on human chondrocytes and forms a heteromeric complex with betaglycan in a ligand and type II TGFbeta receptor independent manner. J Bone Miner Res. 2003;18:289-302.

26. Cheifetz S, Bellon T, Cales C, Vera S, Bernabeu C, Massague J, et al. Endoglin is a component of the transforming growth factor- beta receptor system in human endothelial cells. J Biol Chem. 1992;267:19027-30.

27. Zhao L, Hantash BM. TGF-beta1 regulates differentiation of bone marrow mesenchymal stem cells. Vitam Horm. 2011;87: $127-41$.

28. Chang CB, Han SA, Kim EM, Lee S, Seong SC, Lee MC. Chondrogenic potentials of human synovium-derived cells sorted by specific surface markers. Osteoarthr Cartil. 2013;21:190-9.

29. Leyva-Leyva M, Lopez-Diaz A, Barrera L, Camacho-Morales A, Hernandez-Aguilar F, Carrillo-Casas EM, et al. Differential expression of adhesion-related proteins and MAPK pathways lead to suitable osteoblast differentiation of human mesenchymal stem cells subpopulations. Stem Cells Dev. 2015;24:2577-90.

30. Buhrmann C, Busch F, Shayan P, Shakibaei M. Sirtuin-1 (SIRT1) is required for promoting chondrogenic differentiation of mesenchymal stem cells. J Biol Chem. 2014;289:22048-62.

31. Buhrmann C, Mobasheri A, Matis U, Shakibaei M. Curcumin mediated suppression of nuclear factor-kappaB promotes chondrogenic differentiation of mesenchymal stem cells in a highdensity co-culture microenvironment. Arthritis Res Ther. 2010;12:R127.

32. Caron MM, Emans PJ, Surtel DA, Cremers A, Voncken JW, Welting TJ, et al. Activation of NF-kappaB/p65 facilitates early chondrogenic differentiation during endochondral ossification. PLoS ONE. 2012;7:e33467. 